\title{
Urine Analysis and Ultrasonographic Findings of Dogs Suffering from Renal Failure
}

\author{
Anjali Athaley*, G.R. Bhojne, V.M. Khanolkar, V.M. Dhoot, \\ S.V. Upadhye and C.K. Panchbhai
}

Department of Veterinary Clinical Medicine, Ethics and Jurisprudence, Nagpur Veterinary College, Nagpur, India

*Corresponding author

\begin{tabular}{|c|c|c|}
\hline & \multicolumn{2}{|l|}{ A B S T R A C T } \\
\hline $\begin{array}{l}\text { Ke y w o r d s } \\
\text { Urinalysis, } \\
\text { Ultrasonography, Renal } \\
\text { failure, BUNN, Sr. } \\
\text { Creatinine, Urine culture } \\
\text { examination }\end{array}$ & \multirow{3}{*}{\multicolumn{2}{|c|}{$\begin{array}{l}\text { The study was conducted at TVCC, Nagpur at which a total of } 219 \text { dogs } \\
\text { showed signs of azotemia with BUN level above } 25 \mathrm{mg} / \mathrm{dL} \text { and Serum. } \\
\text { Creatinine level of more than } 2 \mathrm{mg} / \mathrm{dL} \text {. Of these dogs, } 30 \text { dogs were } \\
\text { selected at random and divided into three groups comprising of } 10 \text { dogs } \\
\text { each. Urinalysis was carried out at } 0,18 \text { and } 30 \text { days interval along with } \\
\text { urine culture and Antibiotic Sensitivity tests which were carried out initially } \\
\text { to know the specific drug to be used for the treatment. Ultrasonography was } \\
\text { conducted in all cases to record the different findings in dogs suffering } \\
\text { from renal failure. }\end{array}$}} \\
\hline Article Info & & \\
\hline $\begin{array}{l}\text { Accepted: } \\
\text { 15 September } 2018 \\
\text { Available Online: } \\
10 \text { October } 2018\end{array}$ & & \\
\hline Introduction & & $\begin{array}{l}\text { findings in the urine. Grauer (2007) } \\
\text { proclaimed urinalysis as one of the best }\end{array}$ \\
\hline \multicolumn{2}{|c|}{$\begin{array}{l}\text { Renal failure is one of the leading causes of } \\
\text { deaths in canines. It is life threatening and } \\
\text { always needs immediate treatment and care. } \\
\text { Renal failure accounts to the loss of more than } \\
75 \% \text { of the functional unit of the kidney i.e. } \\
\text { the nephrons. By the time the dogs are } \\
\text { diagnosed, it is often too late to save the dogs. } \\
\text { Various studies are till date being carried out } \\
\text { to minimise the probability of renal failure in } \\
\text { dogs. }\end{array}$} & $\begin{array}{l}\text { sources of detecting renal failure. It helps in } \\
\text { diagnosing if the kidneys are actually } \\
\text { dysfunctioning in its ideal work of filtration } \\
\text { and absorption. When the kidneys are not } \\
\text { functioning properly due to structural or } \\
\text { functional damages, elements like Protein, } \\
\text { Glucose, Ketone bodies etc. escape from the } \\
\text { kidneys into the urine. Proteinuria in dogs } \\
\text { usually occurs due to impaired glomerular } \\
\text { filtration mechanism through which the } \\
\text { proteins that are otherwise not filtered through }\end{array}$ \\
\hline \multicolumn{3}{|c|}{$\begin{array}{l}\text { Urinalysis is regarded as one of the basic tests } \\
\text { used to detect the presence of abnormal }\end{array}$} \\
\hline
\end{tabular}


proteins. Lees et al., (2005) defined the causes of proteinuria into three categories- pre-renal, renal and post-renal. Pre-renal causes included proteinuria due to excess of abnormal plasma proteins that pass through the glomerular wall such as haemoglobin, myoglobin or immunoglobins etc. Renal causes included both functional and pathological causes such as when the disease that was causing proteinuria resolved or when there was structural or functional loss to the kidneys. Post-renal causes included both urinary and extra-urinary causes like excretion of proteins in the urine through exudation or haemorrhaging due to any infection of the urinary system or genitals. Glucosuria is observed when there is significant damage to the proximal renal tubules causing glucose to leak out into the urine or due to the impaired mechanism of tubular reabsorption of the glucose or excess levels of glucose is observed in the blood. Vaden et al., (1997) reported that glucosuria was seen in $23 \%$ of dogs understudy despite of absence of hyperglycemia. Haematuria is a common clinical finding in dogs suffering from uroliths or infections in the urinary tract. Sykes et al., (2010) reported that in dogs suffering Leptospirosis, haematuria and pyuria were amongst the common clinical signs. Ling et al., (2001) conducted a study in dogs suffering from different urinary tract infections. In their study, they found that urine cultures from females yielded more bacterial growth results (37 \%) than in males (29 \%). They also specified on that cystocentesis should be preferred if urine culture needs to be done. Ketonuriais present in cases where the dogs are suffering from starvation, prolonged vomiting and diarrheoa, dietary disturbances etc. The ketone bodies accumulate in the blood when fatty acids are produced from the carbohydrates and then excreted out in the urine. Settles and Schmidt (1994) reported that ketonuria is seen in dogs with impaired tubular reabsorption of the aceto-acetic acid.
The presence of nitrates in urine most commonly implies that there is a bacterial infection in the urinary tract. These bacteria have the enzymes to convert nitrate to nitrites. A small amount of bacteria is regularly present in the urine of the animal. Leucocytes are generally seen in the urine when there is significant amount of inflammation in the urinary tract. This causes infection in the urinary tract to spread causing eventually significant bacteriuria. Assessment of urine specific gravity is considered as an important tool in diagnosing kidney disorders. It helps in understanding the amount of water excreted and if it is being excreted at all.

Use of modern diagnostic modalities like Ultrasonography is a much easier way to diagnose renal failure. It highlights the structural changes in the kidney and urinary system to the point that it can effectively help to diagnose the condition and differentiate between the various disorders that affect the kidneys like acute renal failure, chronic renal failure, cystitis, urolithiasis etc. Tripathi and Mehta (2010) used ultrasonography for the diagnosis of renal disorders in dogs. They reported that structural changes like loss of architectural detail of renal parenchyma, indistinct contours of renal cortex, hyperechoic periphery with reduction in size of kidney, no proper demarcation of cortex and medulla were seen in 4 out of $7 \mathrm{dogs}$ suffering from renal failure whereas Kumar $e t$ al., (2011) found different sonographic findings like 'end-stage' kidney disease (40 $\%)$, hydronephrosis (10\%), nephrocalcinosis $(10 \%)$, renomegaly $(10 \%)$, nephritis $(10 \%)$, nephrolithiasis $(10 \%)$ and renal cyst $(10 \%)$ in the 10 dogs under study. Bhadesiya et al., (2017) conducted ultrasonography on dogs suffering from renal failure to study the structural and morphological changes in the kidneys and found that nephromegaly, indistinct cortico-medullary junction, hypoechoic renal pyramids, hyperechoic renal 
cortex and presence of medullary rim sign were the most common features found in dogs suffering from kidney failure.

\section{Materials and Methods}

Out of the 219 dogs, 30 dogs were selected at random and a complete detailed history, physical and clinical examination was carried out of these 30 dogs.

Urine samples were collected by in a sterile container and sent off for microbial culture examination and Antibiotic Sensitivity test. Urine analysis was conducted using Clintek Urine Chemistry Analyzer at an interval of days 0,18 and 30 to evaluate the changes in the urine constitution of the dogs. Accordingly, treatment was initiated with the specific antibiotic. All the dogs were subjected to ultrasonography using the Toshiba, Justvision 200 ultrasound scanner, with triple frequency transducer having $3.5-5.00 \mathrm{MHz}$ convex and 5.0-7.5 MHz linear transducers.

\section{Results and Discussion}

Out of the 30 study cases, glucosuria was found in $43.33 \%$ of the cases. In the urinalysis conducted on days 18 , the presence of glucose was found to be $16.67 \%$ followed by complete absence of glucose in urine on day 30.Nitrate and ketone bodies both were found in $3.33 \%$ of the dogs each which were completely absent in the urinalysis conducted on subsequent days 18 and 30 respectively which indicated that the animals were recovering with the treatment. Blood was found in $80.56 \%$ of the dogs on day 0 whereas on days 18 and 30, 53.33\% and $33.33 \%$ dogs respectively, presented blood in urine. Bile was found in only $3.33 \%$ dogs followed by none on days 18 and 30 findings of urinalysis (Table 1). Proteinuria was found in the highest amount in $96.67 \%$ dogs. However, on subsequent days 18 and 30,
$86.67 \%$ and $43.33 \%$ dogs exhibited presence of protein in the blood. The proteinuria was studied and its severity was graded as $1+, 2+$ and $3+$. It was found that $20 \%$ dogs exhibited a severity of $1+$, and $16.67 \%$ dogs presented a severity of $3+$ of proteinuria. A severity of $2+$ was found in $60 \%$ of the cases majorly. However, only $3.33 \%$ of the samples showed negativity for protein in the urine (Table 2). On day 0 , all the dogs showed presence of leucocytes in the urine followed by a decline on day $18(66.67 \%)$ and day $30(30 \%)$. A gradual decline was seen in the presence of leucocytes in the urine indicating decrease in the presence of bacteria in the urine too.

Leucocytes were found in the urine ranging from the severity of trace to $3+.6(20 \%)$ dogs each showed leucocytes in the urine each ranging at a severity of trace and 1+. 7 (23.33 $\%)$ dogs presented $2+$ severity and 11 (36.67 $\%)$ dogs exhibited $3+$ severity of leucocyte accumulation in the urine. This gradual decrease in the number of leucocytes could be attributed to the use of the specific antibiotic for which the bacteria are most sensitive to.

The changes in the urine constitution could be attributed to the fact that once the affections were being treated correctly, the kidneys had definite time to heal properly and regulate its function more efficiently.

In the study, the specific gravity of the urine of the dogs suffering from renal failure was also recorded in the urinalysis. $3.33 \%$ cases showed sp.g of 1.005 followed by $30 \%$ dogs exhibiting sp.g of $1.01,33.33 \%$ dogs exhibited sp.g each of 1.015 and 1.02 subsequent urinalysis on day 18 and 30 revealed that the urine specific gravity normalized ranging from 1.001 to 1.015.This normalizing levels could be due to the oral as well as parenteral rehydration done using fluids during the treatment. 
Escherichia coli was found as the dominant organism in $9(33.33 \%)$ dogs followed by Pseudomonas aeruginosa infection in 16.67 $\%$. Staphylococcus aureus was found in 2 $(8.33 \%)$ dogs whereas Klebsiella pneumoniae in $1(2.78 \%)$ dog (Table 3). There was no significant bacteriological growth in 12 (38.89 $\%)$ dogs. Culture and sensitivity tests revealed that the organisms were highly susceptible to Ceftriaxone, Ceftriaxone + Tazobactum, Cefotaxime, Ceftiofur sodium, Cefoperazone + Sulbactum, Ampicillin, Norfloxacin, Nitrofurantoin etc. Accordingly, it was easier to initiate the treatment for the bacterial infection.

The various ultrasonographic findings observed were-

Chronic renal failure- in 12 of 30 cases, loss of cortico-medullary differentiation with no clear margin of the medulla was visible. The loss of architectural landmarks confirmed the chronic condition. Hyperechoic cortex was seen and hyperechoic areas were also noticed at the cortico-medullary junction in few cases. The loss of cortico-medullary differentiation observed during the study could be due to a long standing urinary tract infection which manifested over time due to the owner's negligence or due to the fact that most household dogs relieve themselves in the open where the owner is not able to observe the changes in the urine of their dogs. The findings of the study were consistent with the findings of Kumar et al., (2011) who stated that loss of architectural detail could be a feature of renal diseases and Oburai et al., (2015) who observed reduced corticomedullary differentiation in cases of chronic inflammatory disease or end stage renal disease.

Cystic calculi- Eleven of the cases indicated presence of the gravels from a small to several numbers with different sizes. Numerous small gravels/ calculi were seen in the urinary bladder with urinary bladder wall thickened indicative of cystitis. Cell debris was also seen in all these cases indicating inflammatory changes. Calculi form when the bladder is unable to express completely and in turn calcification of minerals occur. It can also occur due to enlarged prostate, neurogenic bladder or inflammatory changes.

Cystitis- It was the most common feature in almost all of the cases. The urinary bladder wall was thickened with cellular debris within the lumen. It is the most common sequelae of an untreated or ignored urinary tract infection which manifests.

Table.1 Number along with percentage of urinary parameters on days 0,18 and 30

\begin{tabular}{|c|c|c|c|c|c|c|}
\hline & \multicolumn{2}{|c|}{ Day 0 } & \multicolumn{2}{c|}{ Day 18 } & \multicolumn{2}{c|}{ Day 30 } \\
\hline Parameter & $\mathrm{n}$ & $\%$ & $\mathrm{n}$ & $\%$ & $\mathrm{n}$ & $\%$ \\
\hline Glucose & 13 & 43.33 & 5 & 16.67 & 0 & Nil \\
\hline Bile & 1 & 3.33 & 0 & Nil & 0 & Nil \\
\hline Ketone bodies & 3 & 8.33 & 0 & Nil & 0 & Nil \\
\hline Blood & 24 & 80.56 & 16 & 53.33 & 10 & $33.33 \%$ \\
\hline Protein & 29 & 96.67 & 26 & 86.67 & 13 & $43.33 \%$ \\
\hline Nitrate & 3 & 8.33 & 0 & Nil & 0 & Nil \\
\hline Leucocyte & 30 & 100 & 20 & 66.67 & 9 & 30 \\
\hline
\end{tabular}


Table.2 Number along with percentage of proteinuria in dogs suffering from renal failure

\begin{tabular}{|c|}
\hline Severity of proteinuria \\
\hline $1+$ \\
\hline $2+$ \\
\hline $3+$ \\
\hline Negative \\
\hline
\end{tabular}

18

\begin{tabular}{|c|c|}
\hline $\mathbf{n}$ & $\%$ \\
\hline 6 & 20 \\
\hline 18 & 60 \\
\hline 5 & 16.67 \\
\hline 1 & 3.33 \\
\hline
\end{tabular}

Table.3 Number along with percentage of different culture examination in dogs suffering from renal failure

\begin{tabular}{|c|}
\hline Organism \\
\hline Escherichia coli \\
\hline Pseudomonas aeruginosa \\
\hline Staphylococcus aureus \\
\hline Klebsiellapneumoniae \\
\hline No significant growth \\
\hline
\end{tabular}

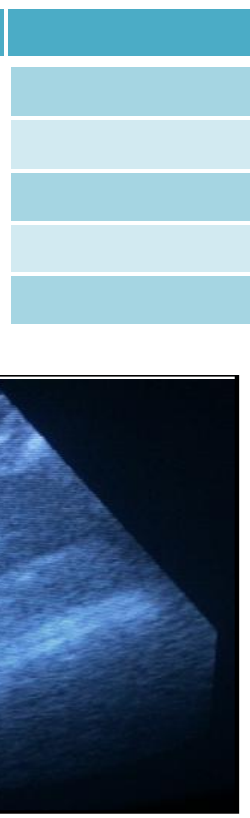

\begin{tabular}{l} 
n \\
9 \\
6 \\
2 \\
1 \\
12 \\
\hline
\end{tabular}

\begin{tabular}{|c|}
\hline$\%$ \\
\hline 33.33 \\
\hline 16.67 \\
\hline 8.33 \\
\hline 2.78 \\
\hline 38.89 \\
\hline
\end{tabular}
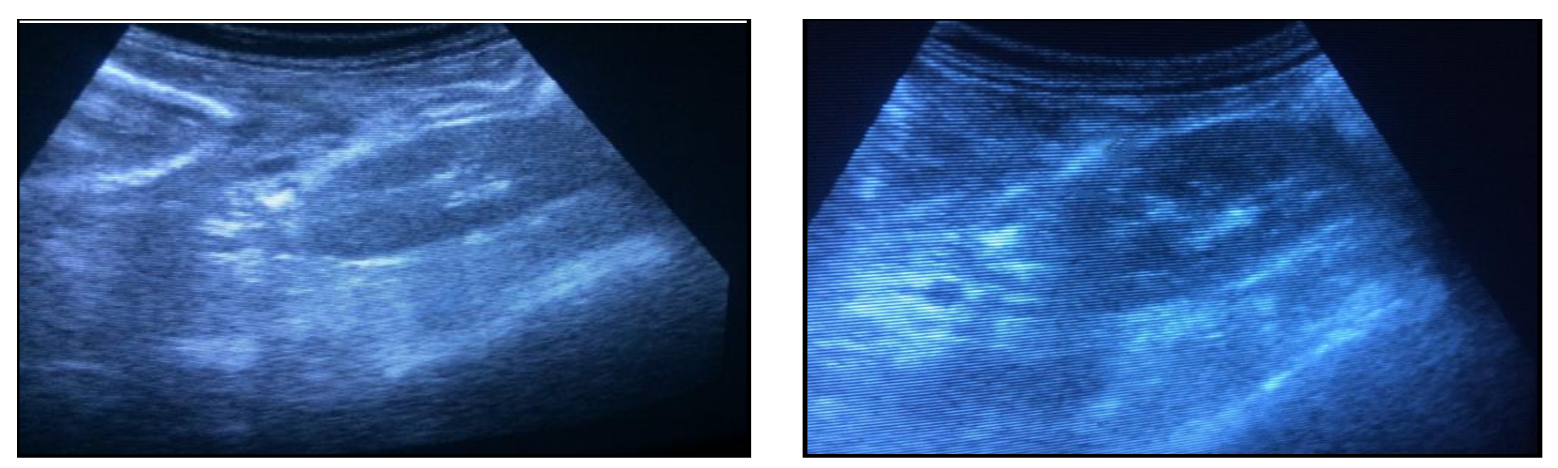

Fig.I Ultrasound indicating loss of corticomedullary differentiation and changes of chronic renal failure

Fig.II Ultrasound indicating loss of corticomedullary differentiation loss at the cranial pole of the kidney indicating to acute renal failure
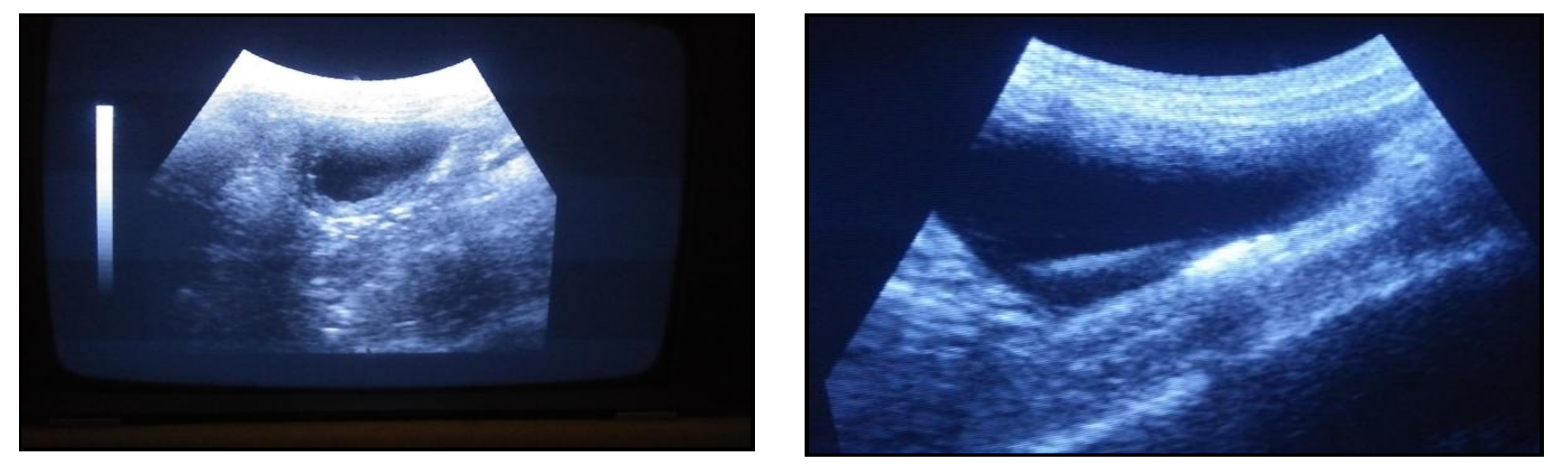

Fig.III Ultrasound indicating thickened wall of urinary bladder along with cellular debris

Fig.IV Ultrasound indicating changes of cystitis with cystic gravels seen 




Fig.V Ultrasound indicating presence of renal calculi

Renal calculi- In one case a renal calculi was noticed. The presence of calculi was seen by its hyperechoic structure and deep acoustic shadow. It was present at the cortex of the left kidney. Renal calculi form when there are more crystal forming substances in the urine like oxalates, uric acid etc.

Hydronephrosis- It was observed in 5 cases. The pelvis seemed dilated with hypoechoic areas and dilated ureters. Presence of uroliths was also confirmed and suspected to have caused the condition. Both unilateral and bilateral hydronephrosis were seen.

Hydronephrosis can occur in the kidneys if there is an obstruction present in the ureters or in the urethra causing disruption in the flow of urine and backflow. The pressure exerted to void urine by the animal causes dilatation of the pelvis of the kidney.

Renal calcinosis- renal calcinosis was observed in 3 cases in the present study. Both bilateral and unilateral calcinosis were seen in the kidneys. Calcinosis was differentiated with areas of hyperechogenecity and shrinking of the medulla. Loss of corticomedullary differentiation was also seen in these cases at places.

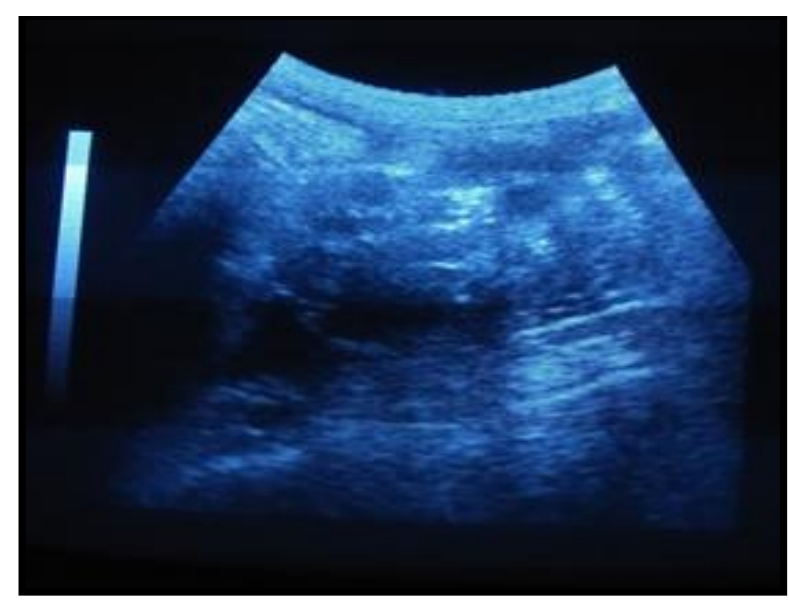

Fig.VI Ultrasound indicating hydronephrosis with change in the shape of the kidney

Renal cyst- renal cyst was noted in the left kidney in one of the cases. A small cavity with smooth outline was seen at the distal pole of the cortex of the left kidney with anechoic lumen present inside.

Pyometra- The condition was observed in 3 cases. The uterus was distended with septalization within the elongated lumen. Anechoic lumen of uterus indicated fluid within the uterus. The kidneys in these dogs showed partial to complete loss of corticomedullary differentiation in one or both the kidneys. Secondary renal failure is known to occur due to the septicimea which occurs due to untreated pyometra.

Prostatic affections- Four cases showed mild to moderate enlargement of the prostate. There was considerable increase in the size of the prostate gland with change in echogenic pattern in the paranchyma in one of the dogs which was also suggestive of a prostatic mass. These prostatic affections can easily obstruct the flow of urine causing further complications.

From the study conducted, it was observed urinalysis and ultrasonography proved to be an effective tool in diagnosing the condition 
and understanding the structural changes in the kidneys in dogs suffering from renal failure.

\section{References}

Bhadesiya, C. M., B. R. Shah, D. R. Mer, P. V. Parikh, N. Rao, A. M. Pande, A. C. Patel, R. G. Jani and S. K. Raval (2017) Studies on Renal Ultrasonography in Azotemic Dogs. Trends in Biosciences. 10(27): 5801-5807.

Grauer, G. (2007) Early diagnosis of chronic kidney disease: Focus on proteinuria, albuminuria and microalbuminuria. Proceedings State of the Art in Renal Disease in Cats \& Dogs. Vetoquinol Academia. pp- 30-35.

Kumar, V., A, Kumar and A. C. Varshney (2011) Ultrasonographic Imaging for structural characterization of renal affections and diagnosis of associated Chronic Renal Failure in 10 dogs. International Scholarly Research Network. pp: 1-11.

Lees, G. E., S. A. Brown, J. Elliot, G. F. Grauer, S. L. Vaden (2005) Assessment and management of proteinuria in dogs and cats : 2004 ACVIM Forum Consensus Statement (Small Animal). J. Vet. Intern. Med. 19: 377-385.
Ling, G. V., C. R. Norris, C. E. Franti, P. H. Eisele, D. L. Johnson, A. L. Ruby and S. S. Jang (2001) Interrelations of Organism Prevalence, Specimen Collection Method, and Host Age, Sex, and Breed among 8,354 Canine Urinary Tract Infections (1969-1995). J. Vet. Intern. Med. 15: 341-347.

Oburai, L. N., V. Rao and B. R. Naik (2015) Clinical and Nephrosonographic Findings in Canine Chronic Renal Failure: A Prospective Study. Journal of Agriculture and Veterinary Science. 8(6): 11-16

Settles, E. L. and D. Schmidt (1994) Fanconi Syndrome in a Labrador Retriever. J. Vet. Intern. Med. 8(6): 390-393.

Sykes, J. E., K. Hartmann, K.F. Lunn, G.E. Moore, R.A. Stoddard, and R.E. Goldstein (2010) 2010 ACVIM Small Animal Consensus Statement on Leptospirosis: Diagnosis, Epidemiology, Treatment, and Prevention. J. Vet. Med. Pp. 1-13.

Tripathi, R. and H. Mehta (2010) Diagnosis of renal disorders in dogs using ultrasound technique. Biosci. Biotech. Res. Comm. 2(2): 213-214.

Vaden, S. L., J. Levin and E. B. Breitschwerdt (1997) A retrospective case-control of acute renal failure in 99 dogs. J. Vet. Intern. Med., 11: 58-64.

\section{How to cite this article:}

Anjali Athaley, G.R. Bhojne, V.M. Khanolkar, V.M. Dhoot, S.V. Upadhye and Panchbhai, C.K. 2018. Urine Analysis and Ultrasonographic Findings of Dogs Suffering from Renal Failure. Int.J.Curr.Microbiol.App.Sci. 7(10): 1971-1977. doi: https://doi.org/10.20546/ijcmas.2018.710.227 